close to $62 \mathrm{kV} / \mathrm{cm}$, considering that the extrapolation was over several orders of magnitude.

\section{REFERENCES}

[1] W. K. Cary, Jr. and J. A. Mazzie, IEEE Trans. Electron Devices, vol. ED-26, p. 1422, 1979.

[2] W. K. Cary, in 2nd Int. Pulsed Power Conf. Digest of Papers, p. $114,1979$.
[3] D. D. Lindberg, R. J. Gripshover, and J. W. Rice, presented at 14 th Modulator Symp., Orlando, FL, June 1980.

[4] P. Felsenthal and J. M. Proud, Phys. Rev., vol. 139, p. A1976, 1965.

[5] J. J. Thomson and G. P. Thomson, Conduction of Electricity through Gases, vol. 2. New York: Dover, 1969 (reprint of 1933 edition).

[6] J. M. Meeks and J. D. Craggs, Electrical Breakdown of Gases. Oxford, England: Clarendon, 1953.

[7] K. R. Allen and K. Phillips, in Proc. Roy. Soc., vol. A278, p. 188 , 1964

\title{
Voltage Recovery Time of Small Spark Gaps
}

\author{
STUART L. MORAN AND LARRY F. RINEHART
}

\begin{abstract}
A two-pulse method is used to determine how fast and to what degree a small spark gap can recover its voltage holdoff capability after breaking down. The first pulse is used to overvolt and break down the gap. The second pulse is used, after a time delay, to determine the voltage recovery of the gap. By varying the time delay to the second pulse, a recovery voltage versus time plot can be obtained. Time delays from $10 \mu$ s to $100 \mathrm{~ms}$ have been recorded. The spark gap discharges millijoules of energy with a gap spacing of less than $1 \mathrm{~mm}$. Recovery has been measured at breakdown voltages of up to $10 \mathrm{kV}$ in argon, hydrogen, and a mixture of the 2 gases. The experimental setup, pulse circuits, and data collection methods are described. Percent voltage recovery versus time plots for various parameters (gas species, gap spacing, and pressure) are discussed.
\end{abstract}

\section{INTRODUCTION}

$\mathbf{M}$ ANY APPLICATIONS of pulse power require high repetition rate spark gap switching. Spark gap recovery time often is the limiting factor in achieving high repetition rates. In order to understand the dominant factors involved in gaseous spark gap recovery, investigations are being made to determine the recovery holdoff voltage as a function of time for small spark gaps. Considerable experimental recovery work has been performed with spark gaps operating at or below atmospheric pressure and at powerline frequencies [1]. The work described herein is directed toward high-pressure gaps which are overvolted by impulses. To obtain recovery information, 2 high-voltage pulses are applied to a spark gap with a variable time delay between them. The first pulse is used to overvolt and break down the gap, and the second pulse is used to determine the voltage holdoff (recovery) of the gap after a time delay. A plot of recovery voltage versus time can be obtained by varying the time delay between the first and second

Manuscript received March 18, 1982; revised July 6, 1982.

The authors are with the Naval Surface Weapons Center, Dahlgren, VA 22448.

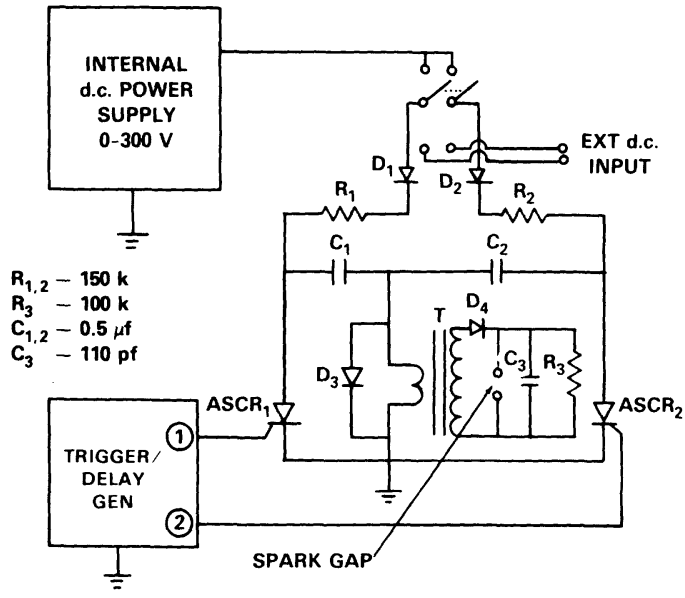

Fig. 1. Schematic of two-pulse circuit.

pulses. This delay can be varied from less than $10 \mu$ s to greater than $100 \mathrm{~ms}$. The time between pulse pairs is about $1 \mathrm{~s}$. The spark gap used for this data is an untriggered pressurized gas spark gap which has approximately Rogowski shaped electrodes $1.3 \mathrm{~cm}$ in diameter. This paper describes the initial effort to determine some of the important parameters and problem areas involved with this type of experiment, as well as the performance characteristics of the experimental setup and procedure.

\section{Two-Pulse Circuit}

The circuit employed to generate the high-voltage pulse pairs is shown in Fig. 1. Capacitors $C_{1}$ and $C_{2}$ are charged through resistors $R_{1}$ and $R_{2}$ by the power supply. A pulse from output 1 of the digital trigger/delay generator turns on the asymmetric silicon controlled rectifier $\left(\mathrm{ASCR}_{1}\right)$ which allows $C_{1}$ to discharge through the primary of the pulse transformer $T$. The secondary winding is connected to be spark gap under test and 


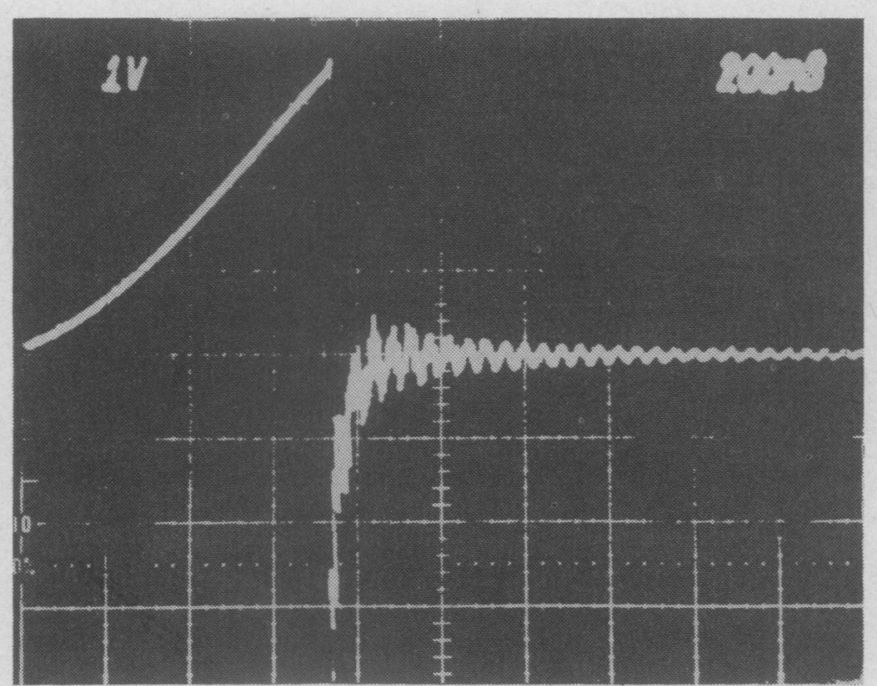

Fig. 2. Voltage waveform across the gap.

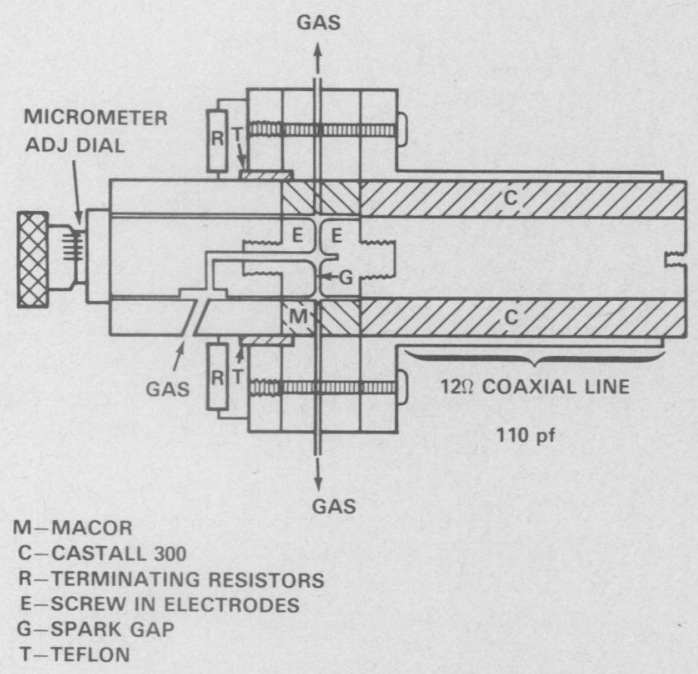

Fig. 3. Cross section of spark gap.

produces a high-voltage pulse which breaks down the gap. After a variable time delay, output 2 of the trigger/delay generator turns on $\mathrm{ASCR}_{2}$ and a second voltage pulse is applied to the gap as $C_{2}$ discharges through the primary of $T$. A small high-voltage capacitor $C_{3}$ is connected directly across the spark gap to provide energy storage for the discharge. Diodes $D_{1}$ and $D_{2}$ isolate $C_{1}$ and $C_{2}$ when a single high-voltage power supply is used. $R_{3}, D_{3}$, and $D_{4}$ damp oscillations in the transformer and spark gap. The values of $C_{1}, C_{2}$, and $C_{3}$ and the transformer characteristics may be varied to change the voltage, energy, and rise time produced across the gap. A typical rate of rise of the voltage across the gap is $5 \mathrm{kV} / \mu \mathrm{s}$, and typical rise times are less than $1 \mu \mathrm{s}$ as shown in Fig. 2.

\section{SPARK GAP DESIGN}

A cross section of the spark gap and its housing is shown in Fig. 3 . It consists of a coaxial structure where the center conductor is broken to form the gap. The impedance of the struc-

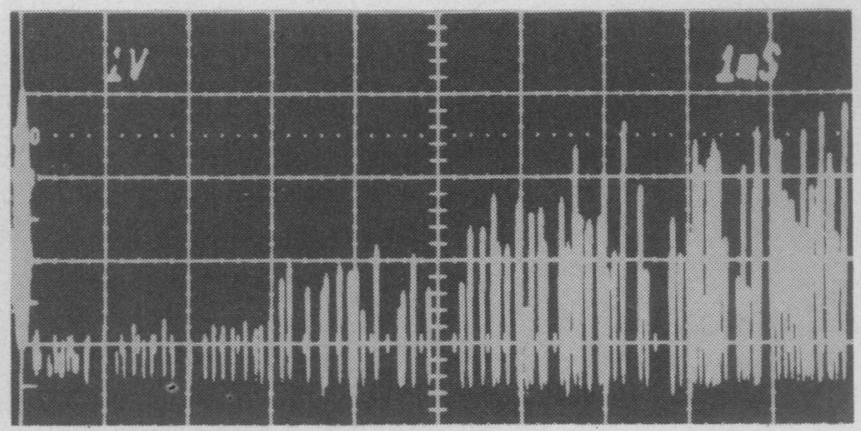

Fig. 4. Swept time delay. First pulse position fixed.

ture is $12 \Omega$ and is terminated in $12 \Omega$ using carbon resistors. The capacitance of the coaxial structure is $110 \mathrm{pF}\left(C_{3}\right.$ in Fig. 2). The inner and outer conductors are copper and the dielectric is Castall 300 , a ceramic-filled epoxy resin. The electrode tips are removable and are made of brass. Surrounding the electrodes is a collar made of Macor, a machineable ceramic. One electrode is adjustable by a micrometer to accurately vary the gap spacing. Before taking data, the electrode surfaces were conditioned by more than 1 million discharges. No attempt was made to resurface the electrodes between experiments with different gases. Gas enters the gap spacing through a hole in the center of 1 electrode and exits through 3 holes in the sides of the gap area. It then passes through a metering valve, a flow meter, and is vented to the atmosphere. The gas flows through the gap at about $0.3 \mathrm{~cm}^{3} / \mathrm{s}$ measured at atmospheric pressure at the vent outlet. Voltage is applied across the coaxial conductors and is measured at the same location with a Tektronix high-voltage probe (P6015). Gap spacing is in the range of $0.1-0.5 \mathrm{~mm}$ and discharges millijoules of energy at several kilovolts.

\section{DAtA Recording Method}

Data are recorded photographically in 2 forms, both of which are multiple-image exposures with an oscilloscope camera. In the first type of photograph, shown in Fig. 4, the scope is triggered on the first pulse of each pulse pair. This places each first pulse trace at the same spot on the left side of the photograph. The time delay to the second pulse is slowly increased for each pulse pair so that a multiple-image photograph is created which shows the breakdown voltage as a function of time delay between pulses. In the second type of photograph, the time delay between pulses is fixed at onehalf the screen width. The position of the traces on the scope is slowly moved halfway across the screen while the shutter is open. This produces the multiple-image photograph shown in Fig. 5, which shows a series of first-pulse breakdowns on the left half of the photo and a series of second-pulse breakdowns on the right half. This indicates the statistical variation of the breakdown voltage of the first and second pulses and provides a statistical comparison of the pulse pairs for one particular time delay. In generating multiple-image photographs, a problem exists because of high intensity blooming of the baseline that tends to obscure the faint voltage pulse images. This 


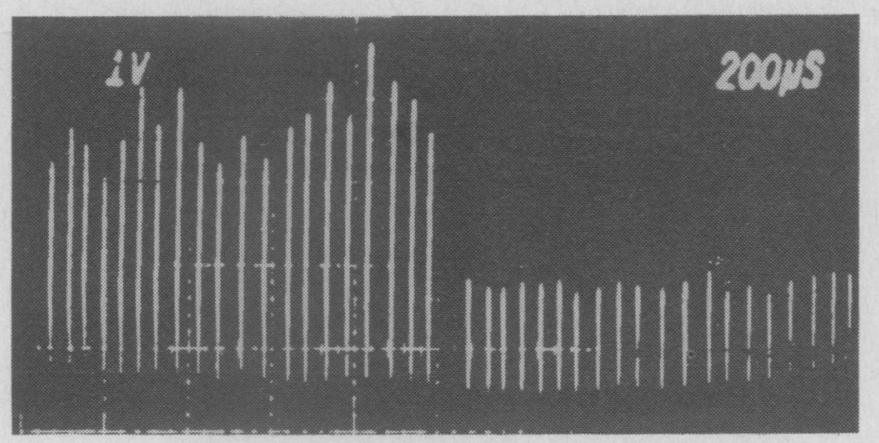

Fig. 5. Swept position. Time delay fixed.

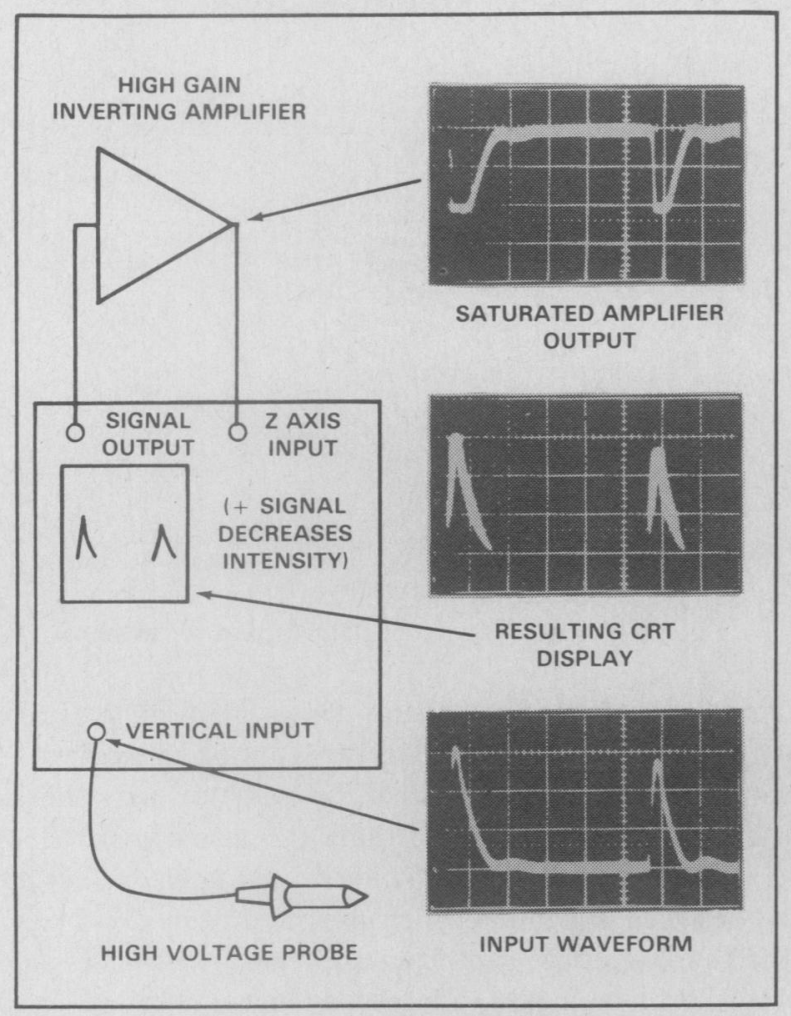

Fig. 6. Baseline blanking scheme.

problem has been solved by using a high gain inverting amplifier to control the intensity of the trace. The original voltage signal is amplified and inverted and is fed back into the intensity ( $Z$ axis) input of the oscilloscope as shown in Fig. 6. This reduces the intensity of the trace at low amplitudes, thereby decreasing or eliminating the baseline.

As is evident from Figs. 4 and 5, there is a significant statistical variation between events. The first- and second-pulse breakdowns, for a given pressure and gap spacing, frequently vary by a factor of 2 . Also, for a given time delay, the ratio of the first-pulse voltage to the second-pulse voltage frequently varies by a factor of 4 . The second-pulse voltage may be even greater than the first. It appears that this variation is due to the statistical time-to-breakdown which allows the rising voltage pulse to fire the gap at different amplitudes and, therefore, to be overvolted to different degrees. In taking data it is, therefore, necessary to do some averaging.

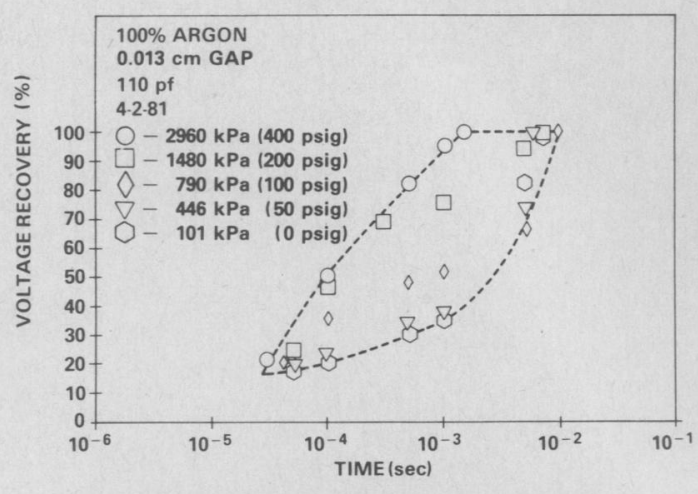

Fig. 7. Percent recovery versus time for argon.

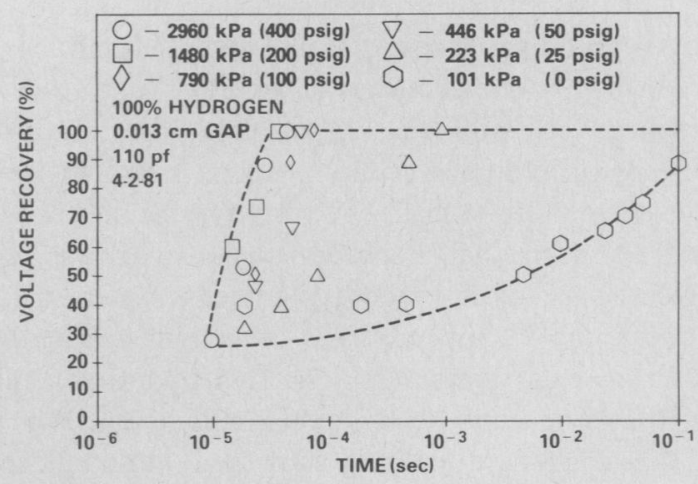

Fig. 8. Percent recovery versus time for hydrogen.

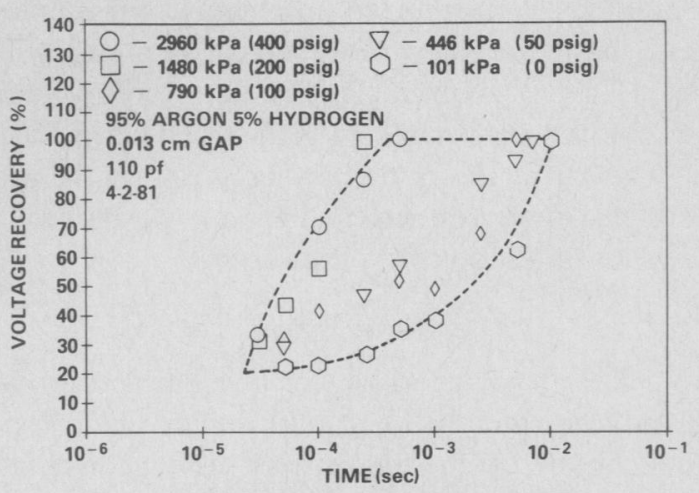

Fig. 9. Percent recovery versus time for hydrogen-argon mix.

\section{EXPERIMENTAL GRAPHS}

Data for the graphs were recorded by choosing a time delay between pulses and making multiple-image photographs similar to Fig. 5. This yields about 30 pulse pairs which are then averaged to give the breakdown and recovery voltage for that time delay, producing one point on the recovery graphs.

Three gases have been tested. They are hydrogen, argon, and a mixture of 95-percent argon and 5-percent hydrogen by volume. Plots of percent recovery versus time are shown in Figs. 7-9. The dotted lines represent the recovery curves for $101 \mathrm{kPa}(0 \mathrm{psig})$ and $2855 \mathrm{kPa}$ (400 psig). Note that for argon, increasing pressure from 0 psig to $2855 \mathrm{kPa}$ (400 psig) decreases recovery time by one order of magnitude. For hydrogen, this change decreases recovery time by three orders of 


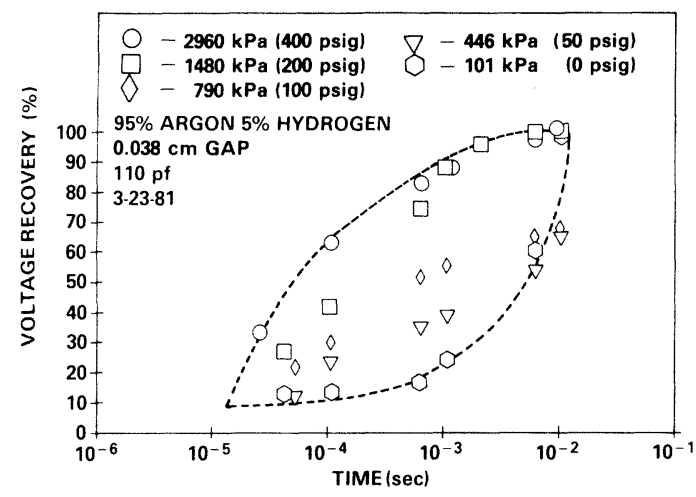

Fig. 10. Percent recovery versus time for hydrogen-argon mix (15-mil gap).

magnitude. Hydrogen recovery is an order of magnitude slower than argon at low pressure, but an order of magnitude faster than argon at high pressure. More recent data indicate that the recovery of hydrogen at low pressure is strongly affected by electrode conditioning. The recovery of the mixture of hydrogen and argon falls between the curves for the separate gases. The shape of the recovery curves is observed to shift from concave up at low pressures to straight or slightly concave down at high pressures. The hydrogen-argon mixture was tested at 2 spacings, $0.013 \mathrm{~cm}$ ( 5 mils) and $0.038 \mathrm{~cm}(15$ mils). The smaller gap spacing showed a weak tendency to recover faster at all pressures (Fig. 10).

The degree of overvoltage was measured by comparing the static breakdown voltages to the average pulse breakdown voltages. Static breakdown Paschen curves are shown in Fig. 11. Fig. 12 shows an example of voltage recovery versus time in terms of the percentage of static breakdown voltage for argon. This curve shows that at low pressures the spark gap is overvolted by several times the static breakdown, but at high pressure almost no overvolting occurs.

\section{CONCLUSION}

This work is an initial effort which will be used to guide the design and construction of a more sophisticated test setup. Some conclusions can be drawn from the experimental results achieved so far. The two-pulse method utilizing a digital trigger/ delay circuit to create a variable time delay between pulses works well, as does the photographic data collection technique using $z$-axis blanking. Gas species and pressure can cause order-of-magnitude changes in recovery times and, therefore,

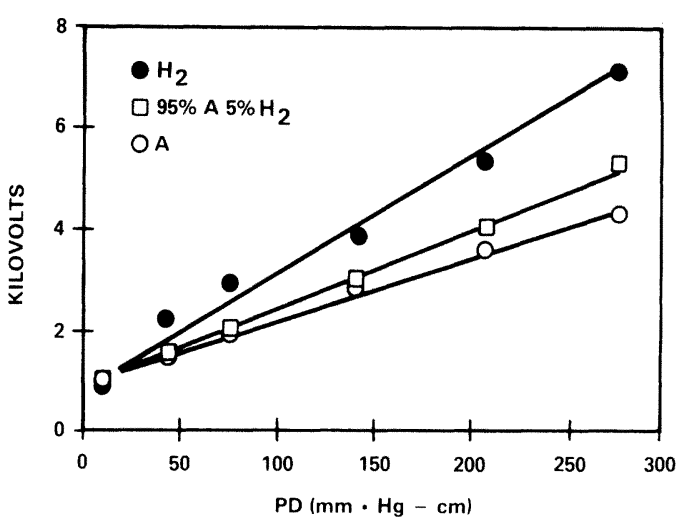

Fig. 11. Static Paschen curves.

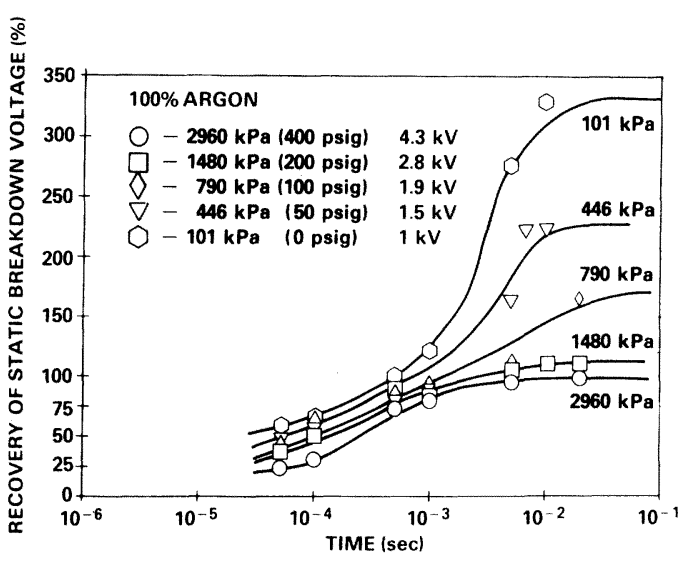

Fig. 12. Percent recovery of static breakdown in argon.

are very important parameters. The statistical variation in time-to-breakdown causes a large variation in breakdown voltage and energy discharged. Since the statistical variations may provide important clues concerning the recovery of the gap, they should be recorded and studied. An upgraded system is being designed and built which uses hydrogen thyratrons to discharge high-voltage capacitors through the gap. This allows shorter rise times, higher voltages, and higher energies. In addition, the amount of energy discharged through the gap can be constant regardless of breakdown voltage. Spark recovery will continue to be studied using this upgraded apparatus.

\section{REFERENCES}

[1] G. A. Farrall and J. D. Cobine, "Recovery strength measurements in arcs from atmospheric pressure to vacuum," IEEE Trans. Power App. Syst., pp. 927-932, Aug. 1967. 